\title{
Effects of temperature on spinodal decomposition and domain growth of liquid-vapor systems with smoothed particle hydrodynamics
}

\author{
Martin Pütz ${ }^{*}$ and Peter Nielaba \\ Universität Konstanz, Fachbereich für Physik, 78457 Konstanz, Germany \\ (Received 22 September 2014; revised manuscript received 5 February 2015; published 5 March 2015)
}

\begin{abstract}
We present a numerical method for simulations of spinodal decomposition of liquid-vapor systems. The results are in excellent agreement with theoretical predictions for all expected time regimes from the initial growth of "homophase fluctuations" up to the inertial hydrodynamics regime. The numerical approach follows a modern formulation of the smoothed particle hydrodynamics method with a van der Waals equation of state and thermal conduction. The dynamics and thermal evolution of instantaneously temperature-quenched systems are investigated. Therefore, we introduce a simple scaling thermostat that allows thermal fluctuations at a constant predicted mean temperature. We find that the initial stage spinodal decomposition is strongly affected by the temperature field. The separated phases react on density changes with a change in temperature. Although, the thermal conduction acts very slowly, thermal deviations are eventually compensated. The domain growth in the late stage of demixing is found to be rather unaffected by thermal fluctuations. We observe a transition from the Lifshitz-Slyozov growth rate with $1 / 3$ exponent to the inertial hydrodynamics regime with a rate of $2 / 3$, only excepted from simulations near the critical point where the liquid droplets are observed to nucleate directly in a spherical shape. The transition between the growth regimes is found to occur earlier for higher initial temperatures. We explain this time dependency with the phase interfaces that become more diffuse and overlap with approaching the critical point. A prolonging behavior of the demixing process is observed and also expected to depend on temperature. It is further found that the observations can excellently explain the growth behavior for pure nonisothermal simulations that are performed without thermostat.
\end{abstract}

DOI: 10.1103/PhysRevE.91.032303

PACS number(s): 64.70.F-, 47.11.-j, 05.70.Ln, 51.20.+d

\section{INTRODUCTION}

The influence of hydrodynamics and temperature on the dynamic process of phase transitions are still open issues. In this work a simulation method for first-order phase transitions of a one-component fluid is introduced. To be more precise, the smoothed particle hydrodynamics (SPH) simulation method is first applied to investigate the dynamics of liquid-vapor phase transitions, especially the so-called spinodal decomposition (SD), where a spontaneous separation occurs after the fluid was instantaneously quenched to a completely unstable state. With our method the whole separation process is in excellent agreement with theoretically predicted properties for all expected time regimes and results from other simulation methods. In addition, SPH evolves the temperature, which is a more realistic approach than most theories that treat the process isothermal.

The separation process splits up into an initial- and a latestage time regime. The predictions for the initial stage concern the growth of long-wavelength "homophase fluctuations" [1], which follows from the Cahn-Hilliard-equation $(\mathrm{CH})$ [2-4], a transition from SD at deep quenches to a rather nucleation-like SD for low quench depths near the critical point $[1,5]$ and an increase of the mean temperature that is due to latent heat observed at nonisothermal simulations [6]. But also in the late stage of phase separation, the domain growth (DG) regime, the SPH results are in agreement with theoretical predictions. The DG process is divided into different growth regimes $[7,8]$ that are characterized by different scaling behaviors of the characteristic length of the domains $\xi(t) \sim t^{\alpha}$. The

*martin.puetz@uni-konstanz.de exponents can be assigned to different growth processes. A diffusion-driven coarsening growth was predicted by Lifshitz and Slyozov (LS) [9] and is expected to yield an exponent of $\alpha=1 / 3$. The influence of hydrodynamics is subdivided in two regimes, where Siggia predicted a crossover from LS to a viscous hydrodynamic regime $(\mathrm{VH})$ with linear scaling $\alpha=1$ [10], followed by Furukawas prediction of an inertial hydrodynamic regime (IH) that corresponds to $\alpha=2 / 3$ [11]. Nevertheless, it must be mentioned that an all-embracing theory is still not found. Many attempts with several simulation methods have been made to prove these predictions (at least partially) [11-20] and to find crossovers between the regimes [21-27]. More recent works take the evolution of temperature into account where a prolongation in the DG regime is observed [6,28]. The SPH method allows us to evolve through all the above-mentioned time regimes in a single simulation, reaching from the early-stage SD to the late-stage DG regimes.

Most of the mesoscopic methods, and so our SPH method, are based on the simple approach for the modeling of phase separation, which is given by van der Waals (vdW) [29] who proposed an equation of state (EOS) for liquid-vapor phase transitions. The vdW theory predicts both, the existence of a completely unstable region, which is enclosed by the spinodal curve, and above that a metastable region enclosed by the coexisting curve (binodal curve) that predicts the coexisting liquid and vapor densities. The spinodal and binodal meet at the so-called critical point. Surface tension arises naturally by the use of the vdW-EOS in SPH and thus interfaces become more diffuse when approaching the critical point. This was first shown by Nugent and Posch [30] and, with a modified vdW-EOS where an additional stress tensor is applied, in a very recent work by Sigalotti et al. [31]. Both studied the droplet formation after an instantaneous volume expansion. In 
this procedure only the mean density of the total volume gets below the spinodal curve, but in fact the dynamical process is only a reshaping of an initial squared (or cubed) liquid droplet to a spherical one. This vdW-SPH approach has been the basis for works on various subjects, such as the oscillation and coalescence of droplets, the determination of the surface tension, or modeling multiphase flow in two [32-37] and recently also in three dimensions [38,39]. Nugent and Posch pointed out that the attractive and repulsive components of the pressure that are given by the vdW-EOS must be treated separately with different smoothing lengths to obtain sensible results. This practice has become a common method. However, from a numerical point of view there is no explanation for this choice and should even be avoided. Furthermore, all of these works have been performed with low resolutions and could not resolve the demixing process at temperatures near the critical point.

The SPH method used in this work is based on the modern formulation of SPH [40,41], which is derived from a Lagrangian and yields very good conservation properties. Physical viscosity is already applied to SPH [40,42] but intentionally not included in our model in order to reduce the complexity. SPH is suitable for very effective parallelization strategies and allows us to perform simulations at very high resolutions in three dimensions. An adequate choice of kernel provides the enlargement of the smoothing range and repeals the requirement of differentiation in long- and short-range components. Instantaneous temperature quenches in the unstable region allow us to observe the growth of "homophase fluctuations" in SD and DG under consideration of hydrodynamics. A thermostat treats the simulation quasiisothermal, because thermal fluctuations at a fixed mean temperature are expected to give a more realistic sight on the separation process of a thermally quenched system than an isothermal treatment. In the context of phase separation, SPH has some advantages over other common simulation methods, like the also mesoscopic Lattice Boltzmann method. It is easy to determine the mean densities and temperatures, to follow their evolution in a phase diagram, and to directly observe the fluid flow.

The paper is structured as follows. In Sec. II the governing equations and the SPH method are presented. In Sec. III first the simulation method and the new scaling thermostat are validated with both static and dynamic test cases. Afterwards, the results of the numerical simulations for the phase separation process without thermostat and with thermostat are presented. The paper is closed by a conclusion in Sec. IV.

\section{METHODS}

In this section the simulation method and the related governing equations are briefly discussed, with focus on recent developments of the code. The SPH method was simultaneously developed by Lucy [43] and Gingold and Monaghan [44], originally for astrophysical purposes (see, e.g., Ref. [45]). It has been extended and further developed until recent days. Therefore, a lot of SPH code implementations are available at present. Today the development also focuses on smaller length scales, which poses further challenges. In the present study a modification of the massively parallel simulation code Gadget2 [46] is used. The modifications comprise an equation of state that supports phase separation, a new kernel interpolant, a thermal conduction equation, and a herein newly developed scaling thermostat.

\section{A. Smoothed particle hydrodynamics}

The SPH method is a mesoscopic mesh-free simulation method where the volume of a fluid is divided in a set of virtual mass elements $m_{i}$ (hereinafter referred to as SPH particles). Every SPH particle is assigned to a volume that is specified by a so-called smoothing length $h_{i}$. These volumes must not be regarded as separated volumes but as rather overlapping each other. Any quantity can be approximated via a weighted interpolation over the neighboring SPH particles. The weighting function $W(\mathbf{r}, h)$, which is also called the smoothing kernel, must satisfy the conditions that $W(\mathbf{r}, h) \rightarrow$ $\delta(\mathbf{r})$ for $h \rightarrow 0, W(\mathbf{r}, h) \geqslant 0$ is monotonically decreasing and twice continuously differentiable [41]. A class of kernels that fulfill these conditions and should further be stable against pairing are the Wendland functions [47]. Instead of common choices, like the class of B-splines or Lucy, we use the Wendland $C_{4}$ kernel function that allows a wider smoothing range. It reads in three dimensions as

$$
W(\mathbf{r}, h)=\frac{495}{32 \pi}(1-r)_{+}^{6}\left(1+6 r+\frac{35}{3} r^{2}\right),
$$

where $r=|\mathbf{r}| / h$ and $(\cdot)_{+}=\max (0, \cdot)$. In practice a typical number of neighbors $N$ determines the smoothing length $h_{i}$ via the estimated density, such that the kernel volume enclose a constant mass [46].

As SPH is a mesh-free method and thus the particles follow directly the flow of the fluid, the hydrodynamic equations can be described in the Lagrangian frame [40]. The governing equations for a heat conducting nonviscous fluid are namely the continuity equation, the momentum equation, and the energy equation, which read as

$$
\begin{gathered}
\frac{d \rho}{d t}=-\rho \nabla \cdot \mathbf{v}, \\
\frac{d \mathbf{v}}{d t}=-\frac{\nabla P}{\rho}, \\
\frac{d u}{d t}=-\frac{P}{\rho} \nabla \cdot \mathbf{v}-\frac{1}{\rho} \nabla \mathbf{j},
\end{gathered}
$$

where $\rho, \mathbf{v}, u, P$, and $\mathbf{j}$ are the density, velocity, specific internal energy, pressure, and heat flux vector, respectively. The equations are solved for each SPH particle. One of the main advantages of SPH is that the total mass of the system is exactly conserved, when the total number of particles does not change within a simulation [41]. The continuity Eq. (2) must not be solved explicitly and can be replaced by the density estimation

$$
\rho_{i}=\sum_{j=1}^{N} m_{j} W_{i j}\left(h_{i}\right)
$$

where $W_{i j}\left(h_{i}\right)=W\left(\mathbf{r}_{i}-\mathbf{r}_{j}, h_{i}\right)$. The resulting density tends to overestimate the correct density. It therefore has to be corrected by a correction term fully described in Ref. [47]. The main 
feature of SPH is that any spatial derivative of a scalar or vector quantity reduces at least to the product of the quantity itself and the derivative of the kernel. Thus, the momentum Eq. (3), with respect to adaptive smoothing lengths, can be derived by a Lagrangian and reads as

$$
\frac{d \mathbf{v}_{i}}{d t}=-\sum_{j}^{N} m_{j}\left[\frac{f_{i} P_{i}}{\rho_{i}^{2}} \nabla_{i} W_{i j}\left(h_{i}\right)+\frac{f_{j} P_{j}}{\rho_{j}^{2}} \nabla_{i} W_{i j}\left(h_{j}\right)\right],
$$

where the $f_{i}$ are correction factors due to the adaptivity of the smoothing lengths and are defined by

$$
f_{i}=\left[1+\frac{h_{i}}{3 \rho_{i}} \frac{d \rho_{i}}{d h_{i}}\right]^{-1} .
$$

The SPH formulation of the first term on the right-hand side of Eq. (4) can easily be expressed in terms of the velocity as

$$
\frac{d u_{i}}{d t}=\frac{f_{i} P_{i}}{\rho_{i}^{2}} \sum_{j}^{N} m_{j}\left(\mathbf{v}_{i}-\mathbf{v}_{j}\right) \cdot \nabla_{i} W_{i j}\left(h_{i}\right) .
$$

The latter thermal conduction term includes a second derivative, since the heat flux vector is given by $\mathbf{j}=-\kappa \nabla T$, with the material specific thermal conductivity $\kappa$. One possible SPH discretization is given by

$$
\frac{d u_{i}}{d t}=\sum_{j=1}^{N} \frac{m_{j}}{\rho_{i} \rho_{j}}\left(\kappa_{i}+\kappa_{j}\right)\left(T_{j}-T_{i}\right) \frac{\mathbf{r}_{i j}}{\left|\mathbf{r}_{i j}\right|^{2}} \cdot \nabla_{i} \bar{W}_{i j},
$$

where $\bar{W}_{i j}=1 / 2\left[W_{i j}\left(h_{i}\right)+W_{i j}\left(h_{j}\right)\right]$ is the arithmetic mean between the two kernel values [48,49]. If the thermal conductivity is treated as a constant, the sum $\left(\kappa_{i}+\kappa_{j}\right)$ in Eq. (9) is replaced by $2 \kappa$. Otherwise, the conduction can be expressed in terms of the density and temperature.

In order to suppress nonphysical behavior at discontinuities, it is necessary to take artificial viscosity into account. A variation of the standard formulation of artificial viscosity [50] is given by

$$
\Pi_{i j}=-\frac{\alpha v_{i j}^{\text {sig }} w_{i j}}{2 \bar{\rho}_{i j}},
$$

where $\alpha$ is called the artificial viscosity constant with a typical value in $[0,1]$. The signal velocity $v_{i j}^{\text {sig }}=c_{i}+c_{j}-3 w_{i j}$ with $w_{i j}=\mathbf{v}_{i j} \cdot \mathbf{r}_{i j} /\left|\mathbf{r}_{i j}\right|$ (only if $\mathbf{v}_{i j} \cdot \mathbf{r}_{i j}<0$, otherwise $w_{i j}=0$ ) and $c_{i}$ is an estimate of the sound velocity. The $\bar{\rho}_{i j}$ denotes the arithmetic mean of the densities due to the particles $i$ and $j$. The implementation is realized by replacing any pressure term $P_{i} / \rho_{i}^{2}$, due to a particle $i$, in Eqs. (6) and (8), by an interparticle term $P_{i} / \rho_{i}^{2}+\Pi_{i j}$ [46]. Note that artificial viscosity is solely implemented for numerical reasons and must not be confused with physical viscosity.

The physical properties of the phase separation process for a one-component fluid can be described by the vdW-EOS. The $\mathrm{vdW}$ theory predicts a fluid to separate into two phases when the fluid undergoes a certain critical point $\left(P_{c}, T_{c}, \rho_{c}\right)$. The separation process is mainly controlled by two components, which can be expressed in terms of the critical point. The first one is a repulsive component and comes up by the assumption of a covolume $\bar{b}=\bar{k}_{b} T_{c} /\left(8 P_{c}\right)$, where $\bar{k}_{b}=k_{b} / \mu m_{p}$ with $k_{b}$ the Boltzmann constant, $\mu$ the mean molecular weight and $m_{p}$ the proton mass. The second component is the cohesive pressure $\bar{a}=27\left(\bar{k}_{b} T_{c}\right)^{2} /\left(64 P_{c}\right)$ and acts ultimately as an attractive force. These considerations result in the mechanical [Eq. (11)] and caloric [Eq. (12)] equations of state for a SPH particle:

$$
\begin{gathered}
P_{i}=\frac{\rho_{i} \bar{k}_{b} T_{i}}{1-\bar{b} \rho_{i}}-\bar{a} \rho_{i}^{2}, \\
u_{i}=\bar{k}_{b} T_{i}-\bar{a} \rho_{i} .
\end{gathered}
$$

Note that Eq. (12) can be used to compute the actual temperature of a particle, which provides that an expansion or compression in terms of the density leads to cooling or heating of the fluid. Furthermore, a minimum internal energy $u_{\min }=-\bar{a} / \bar{b}$ can be derived, since the value of density is not allowed to exceed $1 / \bar{b}$ and $T_{i} \geqslant 0$. The vdW equation had first been applied to SPH in order to study the formation of liquid drops in two dimensions [30]. However, common choices for the smoothing kernel like Lucys kernel always required a separation of Eq. (11) into two components, which severally had to be applied to different smoothing lengths. This differentiation in a long-range attraction and a short-range repulsion was necessary to get sensible results. However, the Wendland kernel from Eq. (1) supports much wider smoothing ranges than the common kernels like Lucys or the $B_{2}$ spline. Hence, there is no need to differentiate attractive and repulsive pressure terms within the formulation of SPH that is used in this work.

The integration in time is done by the generally symplectic leapfrog kick-drift-kick algorithm. The implementation to $\mathrm{SPH}$ is described in Ref. [46]. It is reasonable to use adaptive timesteps in order to reduce the computational effort, which, however, is accompanied by the loss of symplecticity of the integration scheme. In order to minimize errors due to the adaptivity, the timesteps are chosen individually. Thus, the step size of a particle $i$ is set by choosing the minimum timestep of several criteria, but bounded by a maximum step size $\Delta t_{\max }$. The criteria are given by an acceleration criterion, the Courant criterion and a criterion due to temperature flow from Eq. (9). The total timestep condition is given by

$$
\Delta t_{i}=\min \left[\sqrt{\frac{2 \eta_{1}}{\dot{\mathbf{v}}_{i}}}, \frac{\eta_{2} h_{i}}{\hat{v}_{i}^{\mathrm{sig}}}, \frac{\eta_{3}\left(u_{i}-u_{\min }\right)}{(\mathrm{d} / \mathrm{d} t) \tilde{u}_{i}}, \Delta t_{\max }\right],
$$

where $\eta_{1,2,3}$ are integration accuracy parameters and $\hat{v}_{i}^{\mathrm{sig}}=$ $\max _{j}\left[v_{i j}^{\text {sig }}\right]$. The individual timesteps are kept to a binary hierarchy, so that all particles are integrated synchronously at last after the actual existing maximum timestep. As discussed in Ref. [49], energy is barely conserved if particles have unequal timesteps; therefore, Eq. (9) is only integrated at times of synchronization.

\section{B. Scaling thermostat for SPH}

The SPH method, as described above, conserves total energy. Thus, an increase of kinetic energy of the fluid is accompanied with a loss of total internal energy and vice versa. This can be seen as a consequence of the hydrodynamic equations. Furthermore, taking artificial dissipation and thermal conduction into account, where the dynamic process becomes 
irreversible, the total energy is still conserved. Combined with the caloric vdW-EOS Eq. (12), the mean temperature $\bar{T}$ of the system may change as the particle densities diverge within the process of phase separation. When the focus of interest is on the evolution of these separated phases, it might be desirable to keep $\bar{T}$ at a constant desired value $T_{0}$. One way is to keep each temperature $T_{i}$ fixed at $T_{0}$, which would generate an isothermal process, but could not preserve thermal fluctuations within the dynamical system. The two remaining possibilities are either to shift or to scale the mean temperature to the desired value. The former is not reasonable since temperatures below $\bar{T}$ could result in negative temperatures. Therefore, the scaling approach is implemented in the way that the new particle temperatures $\tilde{T}_{i}$ are given by

$$
\widetilde{T}_{i}=T_{i} \frac{T_{0}}{\bar{T}} .
$$

Due to the same conflicts that occur with integrating the conduction term, the synchronization points are also used to update the internal energies by the additional thermal scaling term $\bar{k}_{b}\left(\widetilde{T}_{i}-T_{i}\right)$. It should be noticed that in molecular dynamics (MD), thermostat methods, especially the velocity rescaling method, are used in a very similar way to realize a constant temperature ensemble [51]. Actually, the SPH method, in its simplest nonadaptive formulation, is closely related to MD [52]. The velocity rescaling method for MD does not reflect the proper physical properties of a canonical ensemble and must be extended to more advanced but also common methods, e.g., the Berendsen coupling or NoséHoover thermostat, because it does not allow fluctuations in temperature. However, for SPH the scaling approach is quite suitable because it already treats the temperature as a mesoscopic field quantity. Furthermore, it allows the temperature field to fluctuate around a given mean value.

\section{SIMULATION RESULTS}

In the past decades several approaches have been made in understanding phase separation processes and effects of hydrodynamics on the dynamical process. Therefore, spinodal decomposition (SD) has been studied extensively, both experimentally and theoretically. Since almost all theoretical derivations commonly treat the process isothermal, which is indeed an appropriate approximation for quenches in the neighborhood of the critical point, thermal SD simulations have been performed with a thermal Lattice-Boltzmann (LB) method [6] where the temperature field and thermal conduction were applied. When the SD occurs the mean temperature is found to increase due to latent heat and the domains grow slower than in the isothermal case. However, the thermal fluctuations that arise from compression and expansion play a decisive role for the evolution of deep-quenched systems. This evolution of dynamics is far from clear and shall be focused on in this work from the early stage, where the phases separate, up to the late stage, where the system reaches local equilibrium and is dominated by domain growth and coarsening. The SPH method allows us to observe the thermal evolution for each phase in a direct manner, whereas the thermostat extension allows us to observe fluctuations at a fixed mean temperature.
First the accuracy of the scaling thermostat is demonstrated by both static and dynamic thermal conduction test cases. This is followed by simulations with and without applied thermostat, where the SD and DG regimes are studied and compared with theoretical predictions in detail. The results for various quench depths and applied thermostat are presented in the last part.

The distribution of the initial SPH-particle configuration is realized by a relaxation process. The desired number of SPH particles are uniform randomly distributed in the simulation box before they are integrated in time with an artificial repulsive force term. The particles reach a nearly equilibrated state after a certain number of timesteps. The resulting configuration is a so called glasslike distribution. Note that a small noise in the SPH-density estimation (depending on the number of neighbors $N$ and the initial value of the density $\left.\rho_{0}\right)$ is generated by the nonequidistant spacing of the particles. But in the context of SD a noisy density field is even desired. The simulation box is a cube of side length $L=1$ and the total mass $M=\rho_{0} L^{3}$. Therefore, the mass of one particle is $m_{i}=M / N_{\text {tot }}$, where $N_{\text {tot }}$ is the total number of particles. For a fixed number of neighbors that is set to $N=250$ for all the simulations hereinafter, and an initial density $\rho_{0}=0.5$ the initial standard deviation of the SPH-density is $\sigma_{\rho} \approx$ $5.7 \times 10^{-4}$. The covolume and cohesive pressure are expressed in terms of the critical point $\left(T_{c}, P_{c}, \rho_{c}\right)=(32 / 27,8 / 27,2 / 3)$, which yields $\bar{a}=2.0$ and $\bar{b}=0.5$. Further constant parameters in all simulations are thermal conductivity $\kappa=0.01$, artificial viscosity $\alpha=1.0$, the integration accuracy parameters $\left(\eta_{1}, \eta_{2}, \eta_{3}\right)=(0.0025,0.15,0.1)$, and $\Delta t_{\max }=10^{-4}$. Periodic boundary conditions are applied, since boundary effects shall be neglected here.

\section{A. Validation of the scaling thermostat approach}

The SPH implementation of the thermal conduction equation with the new approach for a scaling thermostat have not yet been applied to the vdW-EOS. In order to evaluate the performance and accuracy of these, a validation test case is performed with the four resolutions of $10^{4}, 5 \times 10^{4}, 10^{5}$, and $10^{6} \mathrm{SPH}$-particles. We consider a planar thermal interface of a homogeneous liquid phase with the initial density $\rho_{0}=1.5$. The interface is located at $x_{m}=0.5$ in $x$ direction and separates the initial temperatures of $T_{l}=1.0$ and $T_{r}=0.8$, where the indices $r$ and $l$ denote the right and the left half of the box, respectively. This initial configuration is purposefully chosen to be out of the binodal region. Hence, thermal effects due to phase separation are neglected.

First we are interested in the capability of the thermal conduction to capture a test case, which can be analytically solved $[49,53]$. That is the initial configuration described above, but concerning a fluid at rest. To meet this, the velocities are kept fixed at zero. The analytic solution for the temperature is given by an error function term,

$$
T(x, t)=\bar{T}+\frac{\Delta T}{2} \operatorname{erf}\left(\frac{x-x_{m}}{2 \sqrt{\chi t}}\right),
$$

where $\Delta T=\left|T_{l}-T_{r}\right|$ and $\chi=\kappa / \rho_{0} c_{p}$ is called thermal diffusivity with $c_{p}=1$. The internal energy is related to the temperature by Eq. (12). Therefore, the internal energy 

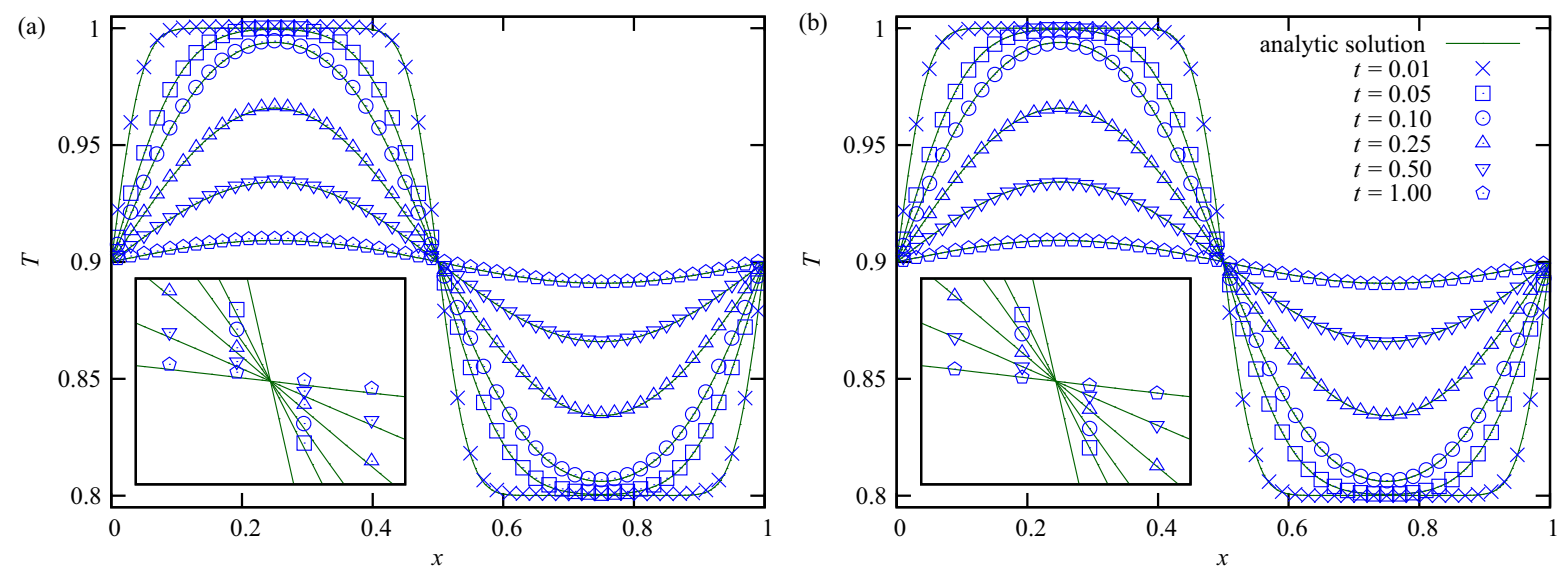

FIG. 1. (Color online) Time sequences of the temperature field $T$ of a resting fluid with an initial thermal discontinuity at $x_{m}=0.5$ with $10^{6} \mathrm{SPH}$-particles and periodic boundary conditions. While in panel (a) the pure thermal conduction equation is used, in panel (b) the thermal conduction equation and scaling thermostat are applied. The symbols represent the simulation results. The corresponding analytic solutions are drawn by the solid lines. The inset boxes show enlargements of the central region of size $\left(x_{m} \pm 0.04, \bar{T} \pm 0.015\right)$.

solution is also given by an error function term that is similar to Eq. (15). This relation is mainly given by the densityweighted cohesive pressure term, which causes a noise at the conversion from temperatures to internal energies, when the glasslike distribution is used for the initial particle positions. One of the interesting features of the thermal conduction term is that those noisy temperature differences are instantly smoothed out. That is why the internal energies are used for initialization instead of the temperatures. Note that this procedure does not have any effect on the mean values of the quantities but on single-particle values. As periodic boundaries are applied, they must be taken into account in the analytic solution. Figure 1 shows a comparison of the temperature profiles from the simulation results with $10^{6} \mathrm{SPH}$ particles at six different times of the pure thermal conduction equation [Fig. 1(a)] and with the additionally applied scaling thermostat [Fig. 1(b)]. The inset boxes show the enlarged central regions. The symbols represent the simulation results and are obtained via a histogram calculation with 50 bins in $x$ direction.
Both simulations reproduce the analytic solution well, but a closer look at the inset boxes in Fig. 1 reveals that the simulation with pure thermal conduction tends to overestimate the temperature values not only in the central region but in the whole simulation volume. This can be addressed to the kernel interpolation procedure that produces density estimations that are slightly too high. However, this incorrect behavior is compensated by the scaling thermostat, which almost perfectly predicts the temperature values expected from the analytic solution. In order to verify the functionality of the scaling thermostat in dynamic systems the same initial configuration is used but with released particles. In this case the analytic solution is expected to hold only a short period of time, which is just as long as the density can be considered to be approximately homogeneous. In addition, these density changes that are due to the particle movement (and therefore the fluid flow) repeal the similarity between the evolution of the internal energy and the temperature. In Fig. 2 the evolution of the densities, temperatures, and internal energies for the four different resolutions with applied thermostat are

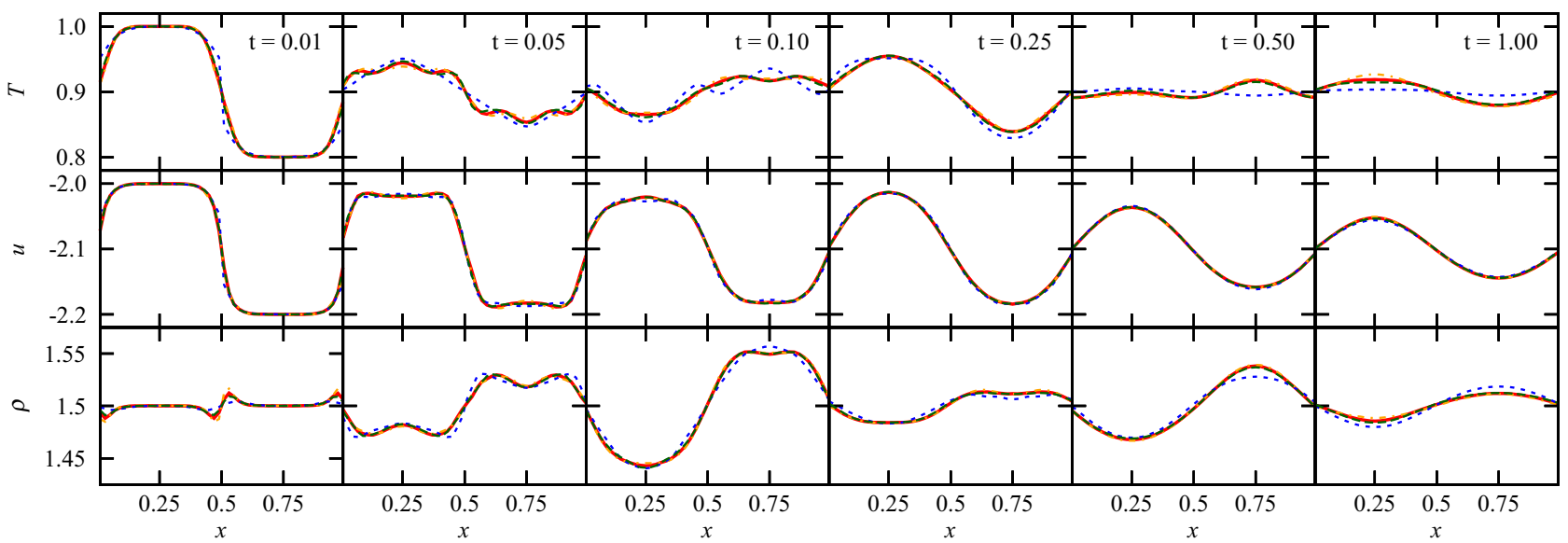

FIG. 2. (Color online) Time sequences of the temperatures $T$, internal energies $u$, and densities $\rho$, for the calculations with thermostat. The different lines and colors represent the results for the resolutions $10^{4}$ (short-dashed blue line), $5 \times 10^{4}$ (long-dashed green line), $10^{5}$ (solid red line), and $10^{6}$ (dashed-dotted orange line). 


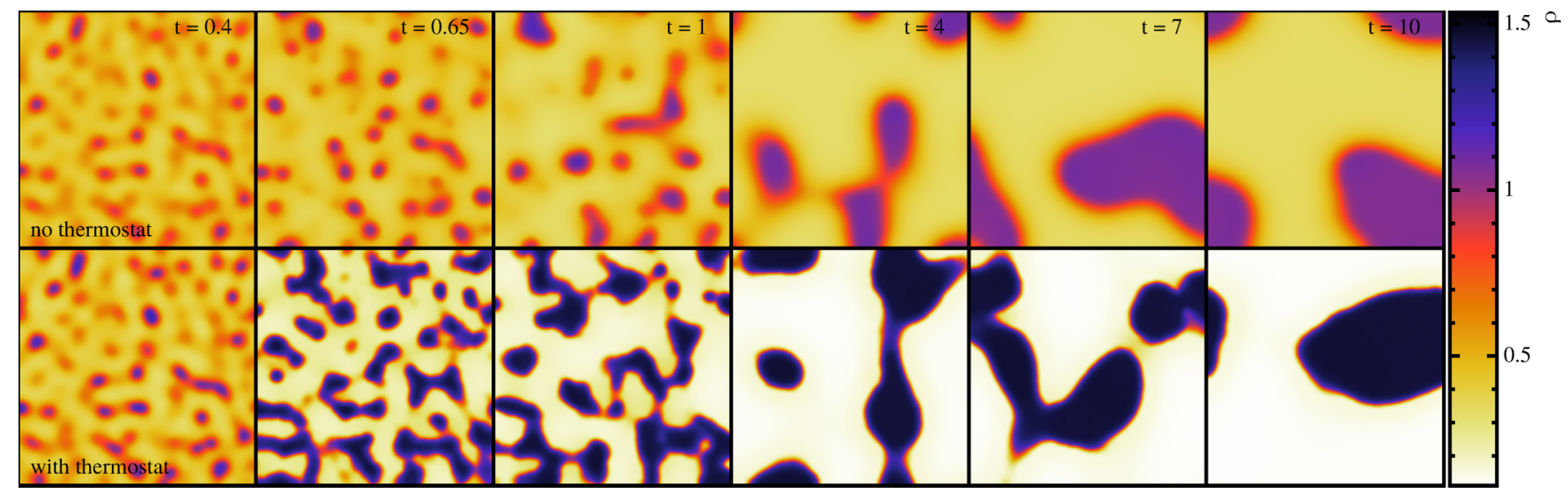

FIG. 3. (Color online) Comparison of density rendered cross-section slices from simulation snapshots at the times $t=0.4,0.65,1.00,4.00$, 7.00, and 10.00. The pure thermal simulations with no thermostat (top row) and with thermostat (bottom row), with a side length of the box size $L=1$. Note that even $t=10.00$ is far from equilibrium and the actual shapes of the three-dimensional structures cannot be determined in the two-dimensional representation.

shown. Note that the simulations have also been performed without the scaling thermostat, but they only differ in the poorer temperature estimation as it was observed in the nondynamical case. The comparison reveals a limited ability in capturing this dynamic test case. The simulations with higher resolutions produce very similar results, whereas the lowest resolved simulation with $10^{4}$ particles differ significantly from these. Therefore, the lowest resolution cannot be expected to adequately describe the dynamics of a vdW fluid. The evolution of the internal energies still have the error-functional shape very similar to the analytic solution in the nondynamical test case. However, the effects of the dynamics can be seen in the density field that fluctuates between high and low densities. It is driven by the actual present temperature. Due to the balance between computational expense and reasonably good results the resolution of $10^{5}$ particles is chosen for all further simulations.

\section{B. Spinodal decomposition and domain growth}

\section{Effect of the thermostat}

The effects of the thermostat on the phase separation process are studied on the basis of two simulations with $N=10^{5}$ particles. They only differ in applying the thermostat. Corresponding to an instantaneous deep quench further initial values are set to $T_{0}=0.8$ and $\rho_{0}=0.5$ for both of the simulations. This is below the spinodal curve and therefore completely unstable, such that the system is expected to immediately initiate to separate. In this initial stage several density peaks, due to the initial density noise, grow in height with time, which is often called "homophase fluctuations" [1]. This is almost identical for both of the simulations. Indeed, the point in time of demixing $\tau_{0}$ (defined as the point where a particle density first exceeds $\rho_{c}$ ) is the same $\tau_{0}=0.31$. However, from this moment on the evolution of the simulations differ significantly. Figure 3 shows cross-section slices of the density of both simulations at different times. The simulation without thermostat forms a wider and more diffuse interface between the two phases than the one with thermostat. The critical density is used as a threshold value $\rho_{\text {th }}=\rho_{c}$ to distinguish between the liquid or vapor phase and to calculate the mean temperature per phase. In Fig. 4(a) a comparison of the walk through the temperature-density diagram is shown. The density values are obtained by a median calculation for each phase. The median is chosen instead of more common choices, such as the arithmetic mean or the modus for the reason that these are very susceptible to the formation of diffuse interfaces at higher temperatures in the initial stage. In
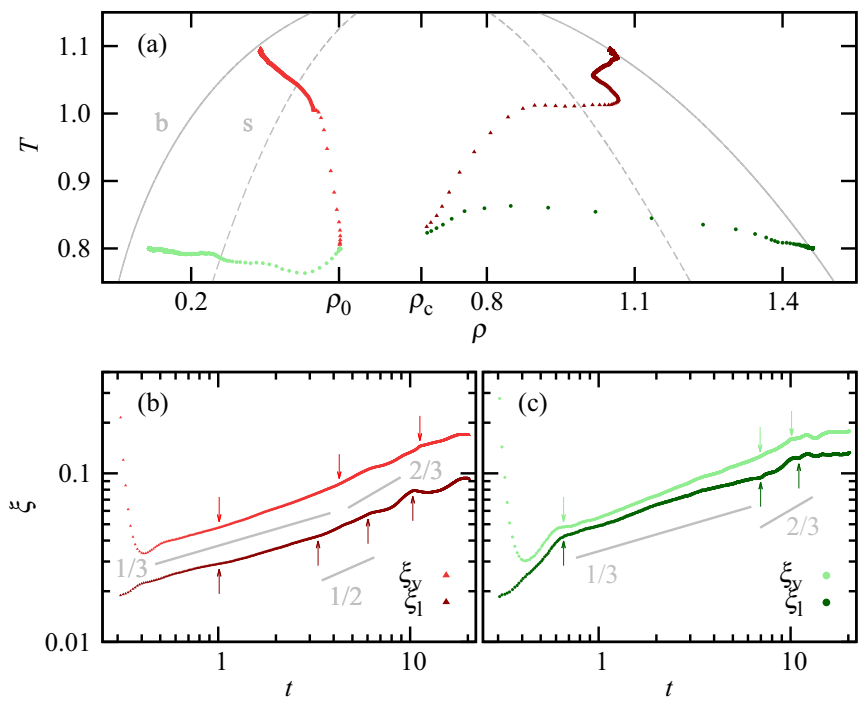

FIG. 4. (Color online) (a) Temperature-density diagram at equidistant timesteps with $\Delta t=0.01$ up to $t=20$ for the $T_{0}=0.8$ and $\rho_{0}=0.5$ simulations without thermostat (red triangles) and with applied thermostat (green dots). The dark and light colors refer to the liquid and vapor phase, respectively. The gray lines represent the binodal (solid line) and spinodal (dashed line) curves from vdW theory and Maxwell construction. The density values are obtained via median and the temperatures are arithmetic means of each phase. The characteristic lengths of the vapor $\xi_{v}$ and the liquid phase $\xi_{l}$ for the same simulations are shown in panels (b) and (c), respectively. The arrows point fit intervals and the gray lines refer to slopes of $1 / 3$ and $2 / 3$ to guide the eye. 
the late stage, the density distribution shows two sharp peaks in the histogram and the three types of means differ only slightly. But in the early stage the robustness of the median to single strong-density deviations improves the understanding of the separation process. The points in Fig. 4(a) mark equidistant timesteps. It shows that the early stage of the demixing process lasts longer in the pure thermal case. Associated therewith the thermal heating is an important difference to the thermostat case in this early stage. This effect has recently been shown by Gan et al. [6] who implemented a thermal model for phase separation to LB simulations. In this case, theoretical predictions are not clear. However, the SPH method provides a direct view on the evolution of the phases, which reveals that in the absence of a thermostat both phases heat up. More precisely the SD process can be divided into the three following parts. The aforementioned heating is followed by a time interval where the heating stagnates and only the liquid density is further approaching the coexistence density. Each of these parts last about $\Delta t \approx 0.1$. In the third part, which lasts longer by two orders of magnitude, temperature is raised again. But the liquid density shows an oscillating behavior until it reaches the coexistence curve. On the other hand, with applying a thermostat the interesting effects are seen on the vapor phase. Instead of a temperature increase, as observed and expected from the pure thermal simulation, the vapor temperature first decreases alongside with a density decrease. This process is followed by a slow temperature increase approaching both the predicted temperature and coexistence density. A contrary evolution is observed for the liquid phase where a density increase is accompanied with an increase in temperature.

Anyhow, the heating and cooling behavior of both simulations can be explained by the caloric EOS and thermal conduction in Eq. (4). The initial density noise is intensified by the gradient of Eq. (11). These density differences are coupled to the temperature by Eq. (12). In the pure thermal case the mass of the liquid phase dominates the simulation volume. With its immense temperature increase the vapor phase is heated via thermal conduction. Therefore, the initial $\rho_{0}$ and $T_{0}$, which determine the mass fraction of the separated system, predict the equilibrated mean temperature. With a high gas-to-liquid mass fraction the system should decrease in temperature, whereas a low gas-to-liquid fraction leads to heating, as observed here. Note that the vapor temperature decrease that is always observed in simulations with applied thermostat should also be observed in simulations without thermostat for quenches close to $T_{c}$, where the heating of the liquid phase is minimally extensive.

The late stage of phase separation is dominated by the domain growth and coalescence of droplets. It is quantified by the scaling behavior of the characteristic length scales of the domains, i.e., $\xi(t) \sim t^{\alpha}$, where $\alpha$ depends on the time regime. Conventionally, $\xi(t)$ can be obtained via several methods, such as the structure factor or a two-point correlation function [1]. However, there are several reasons to define $\xi(t)$ in a different way. The calculation of a structure factor goes along with a Fourier transformation and, therefore, requires a regular grid. Also, the maximum value that can be obtained via two-point correlation is half the box size, even though the domain sizes in our simulations exceed this value. Therefore, in this work $\xi(t)$ is calculated straightforward as the mean of the minimum distances from one phase to the other. There are several advantages of this calculation method. The resolution does not depend on the grid sizes, which must be chosen for the Fourier transformations or histogram calculations. In addition, it allows us to distinguish between liquid and vapor phase, thus, the lengths can be treated separately. The characteristic length, e.g., for the liquid phase, is then defined as the arithmetic mean of the particlewise calculated minimum distance to the vapor phase,

$$
\xi_{l}(t)=\frac{1}{N_{l}} \sum_{i_{l}}^{N_{l}} \min _{j_{v}}\left(\left|\mathbf{r}_{i_{l}}(t)-\mathbf{r}_{j_{v}}(t)\right|\right),
$$

where $i_{l}$ and $j_{v}$ belong to the liquid and vapor phase, respectively, and $N_{l}$ is the total number of liquid particles. This method is actually very robust and allows us to trace the phase separation process even until the very late stage, where the coalescing process is almost completed and only the single separated domains remain in local equilibrium. Note that the values obtained by Eq. (16) are smaller compared to conventional methods; e.g., for a spherical-shaped droplet $\xi_{l}$ would be $1 / 4$ of the diameter of the sphere. Calculations of the characteristic lengths of vapor $\xi_{v}$ and liquid $\xi_{l}$ for both simulations are presented in Figs. 4(b) and 4(c). The comparison between the two simulations reveal only slightly different behavior. The already mentioned initial stage "homophase fluctuations" exceed $\rho_{c}$ in both cases and end up with crossing the spinodal curve. This is were the liquid curves $\xi_{l}$ show a slight kink from where the growth process is dominated by the LS mechanism with $\alpha=1 / 3$. In both simulations the very late stage that is dominated by inertial hydrodynamics $(\mathrm{IH})$ with $\alpha=2 / 3$ is observed. One of the main differences is that the pure thermal simulation exhibits a regime with $\alpha=1 / 2$ between these two growth rates. This may be understood as a transition regime where LS mechanism is overlaid by IH effects. Regarding the higher temperature of this simulation it also shows that the regime of hydrodynamics is not temporally fixed and may depend on temperature. Nevertheless, in the simulation with thermostat the two regimes can clearly be distinguished and identified. Note that the model does not include physical viscosity and, therefore, it is not expected to observe the intermediate introductorily described viscous hydrodynamic regime. In Table I the results for the exponents from a least-square regression analysis to the corresponding fit intervals from Fig. 4 are presented. Note that the intervals are only suggestions by the authors based on the shapes of the curves and the evaluated exponents may vary within rearranged intervals. But it should further be mentioned that the fitted $\alpha_{l}$ in the suggested arrangement of intervals are in very good agreement with the theoretical predictions for both types of simulations $[10,11]$. The $1 / 2$ exponent in a thermal, in contrast to the $2 / 3$ exponents for an isothermal system, was also found with LB simulations [6].

The necessity to treat liquid and vapor separated becomes clear by comparing the characteristic lengths for the vapor $\xi_{v}$ with the liquid curves. It is due to the initial state with $\rho_{0} \leqslant \rho_{c}$ that $\xi_{v}=1$ at the initial state. The increase of "homophase fluctuations" arise from the vapor state and exceed $\rho_{c}$. Thus, the nuclei are only observed in the liquid phase. From the vapor point of view, $\xi_{v}$ decreases with the increasing number of 
TABLE I. The exponents $\alpha$ (with asymptotic standard error ${ }^{\mathrm{a}} \delta \alpha$ ) are least-square fitted within the fit intervals suggested in Fig. 4 . The abbreviation nt and wt denote the simulations "no thermostat" [Fig. 4(b)] and "with thermostat" [Fig. 4(c)].

\begin{tabular}{lcccr}
\hline \hline Simulation & Time & $\alpha_{l} \pm \delta \alpha_{l}$ & Time & $\alpha_{v} \pm \delta \alpha_{v}$ \\
\hline $\mathrm{nt}$ & $1.00-3.30$ & $0.333 \pm 0.001$ & $1.00-4.30$ & $0.419 \pm 0.001$ \\
& $3.31-6.00$ & $0.509 \pm 0.001$ & $4.31-11.3$ & $0.502 \pm 0.002$ \\
wt & $6.01-10.3$ & $0.667 \pm 0.008$ & - & - \\
& $0.65-7.00$ & $0.329 \pm 0.001$ & $0.65-7.00$ & $0.424 \pm 0.001$ \\
& $7.01-10.9$ & $0.671 \pm 0.006$ & $7.01-9.90$ & $0.621 \pm 0.004$ \\
\hline \hline
\end{tabular}

${ }^{\mathrm{a}}$ Asymptotic standard error expresses the accuracy in accordance to the calculated characteristic lengths.

liquid nuclei and further undergoes a minimum value. Contrary to the intuitive expectation this minimum does not coincide in time with the maximum number of nuclei. Furthermore, it points out that the nuclei combine and build a fine "spongelike" structure until it is totally interconnected to one single domain. All beforehand the minimum of $\xi_{v}$ is reached (in further calculations this point in time is defined as $\tau_{1}$ ). As this cannot be expected by the cross-section slices in Fig. 3, this fluid motion and structure formation is only observable in three dimensions. The coarsening of the liquid domain can also be observed in the fast increase of $\xi_{v}$ after $\tau_{1}$. So far in both simulations the $\xi_{v}$ are very similar and also in the following stage of growth a scaling law with $\alpha_{v} \approx 0.42$ is observed as depicted in Table I. Only in the very late stage the growth rates differ. The simulation without thermostat shows a rate of $\alpha_{v}=1 / 2$, whereas the simulation with thermostat shows a faster growth of $\alpha_{v} \approx 0.62$. The difference is due to the differing late-stage mean temperatures. But also in accordance with the growth rates of the liquid domains at the very late stage.

It seems only meaningful to compare the initial stage to the thermostat simulation, because of the temperature increase that is due to latent heat in the pure thermal simulation. The mean equilibration temperature at $t=20$ for the latter one is $T_{\mathrm{wt}} \approx 0.8$ compared with $T_{\mathrm{nt}} \approx 1.1$. The equilibration temperature for simulations without thermostat seems hardly predictable. Possible dependencies to the initial parameters are the density, the wavelength and amplitude of the density noise, the temperature $[15,18]$, heat conduction, and in the case of a nonideal fluid, the viscosity [28]. In the next part the quench depth is varied with applied thermostat to improve the understanding of the phase separation process, with special attention to the temperature evolution and thermal fluctuations. This provides one of the main features of the SPH method in contrast to other fully isothermal approaches.

\section{Quench depth variation with thermostat}

Simulations with different quench depths are performed in order to compare the dynamic behavior and the growth rates. The quench depth means the relative distance from the critical point $1-T_{0} / T_{c}$. We focus primarily on the late stage, where the demixing process is almost completed and the domains are growing. Therefore, various initial temperatures, ranging from a deep-quenched $T_{0}=0.6$ to low-quenched $T_{0}=1.1$, are set. The simulations are all scaled by the thermostat. The initial density is independent from the quench depth set to $\rho_{0}=0.5$. The total simulation time ranges from $\approx 20$ to $\approx 50$, depending on the dynamics of the system in order to simulate up to a time where finite-size effects influence the dynamics. Note that the simulations are not evolved until the total equilibration long-time limit is reached, which is mainly due to the absence of physical viscosity in our model. Exemplarily a representative set of simulations is chosen of which crosssection slices of the density in a temporal evolution are shown in Fig. 6. An interesting correlation is observed. The evolutions of the simulations depend significantly on the temperature. The initial fluctuations that are expected to finally form the liquid phase arise much slower with decreasing the quench depth. This is also supported by a comparison of the total kinetic energies $E_{\text {kin }}=\sum m_{i} v_{i}^{2} / 2$ (shown in Fig. 5). The maximum value of kinetic energy is located between $\tau_{0}$ and $\tau_{1}$ and decreases with increasing $T_{0}$. A further look on the inset box reveals that for the simulation with $T_{0}=1.1$ local equilibrium could not be reached within the simulation time and the kinetic energy is even growing at $t=50$. Therefore, this simulation is considered separately later on. A qualitative comparison between the remaining simulations regarding the arise of "homophase fluctuations" and the formation of "spongelike" structures in the initial stage reveals a very similar growth behavior even though the temporal evolutions differ. However,

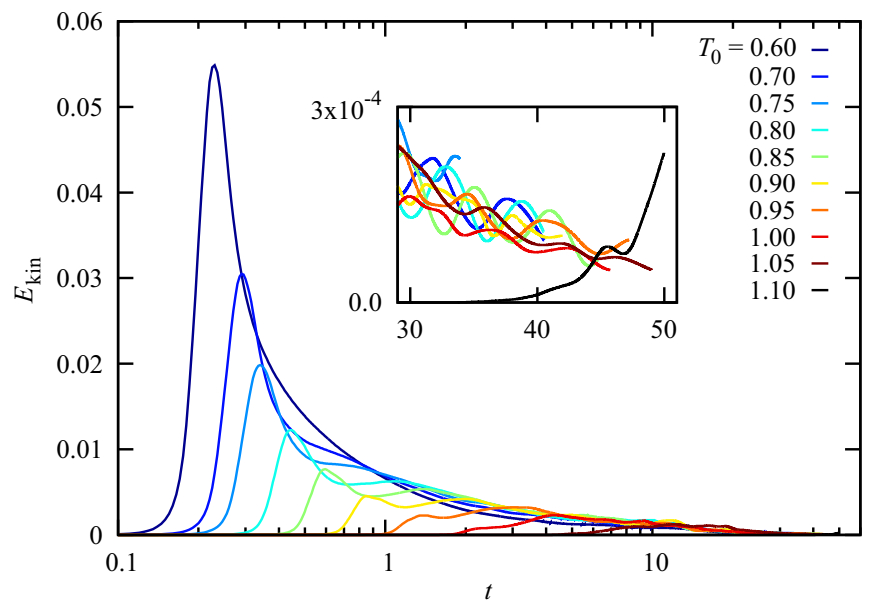

FIG. 5. (Color online) Temporal evolution of the kinetic energies for the temperatures $T_{0}=0.60,0.70,0.75,0.80,0.85,0.90,0.95$, $1.00,1.05$, and 1.10 (from left to right). The inset box shows an enlargement of the curves in the main box at the end of simulation times. 


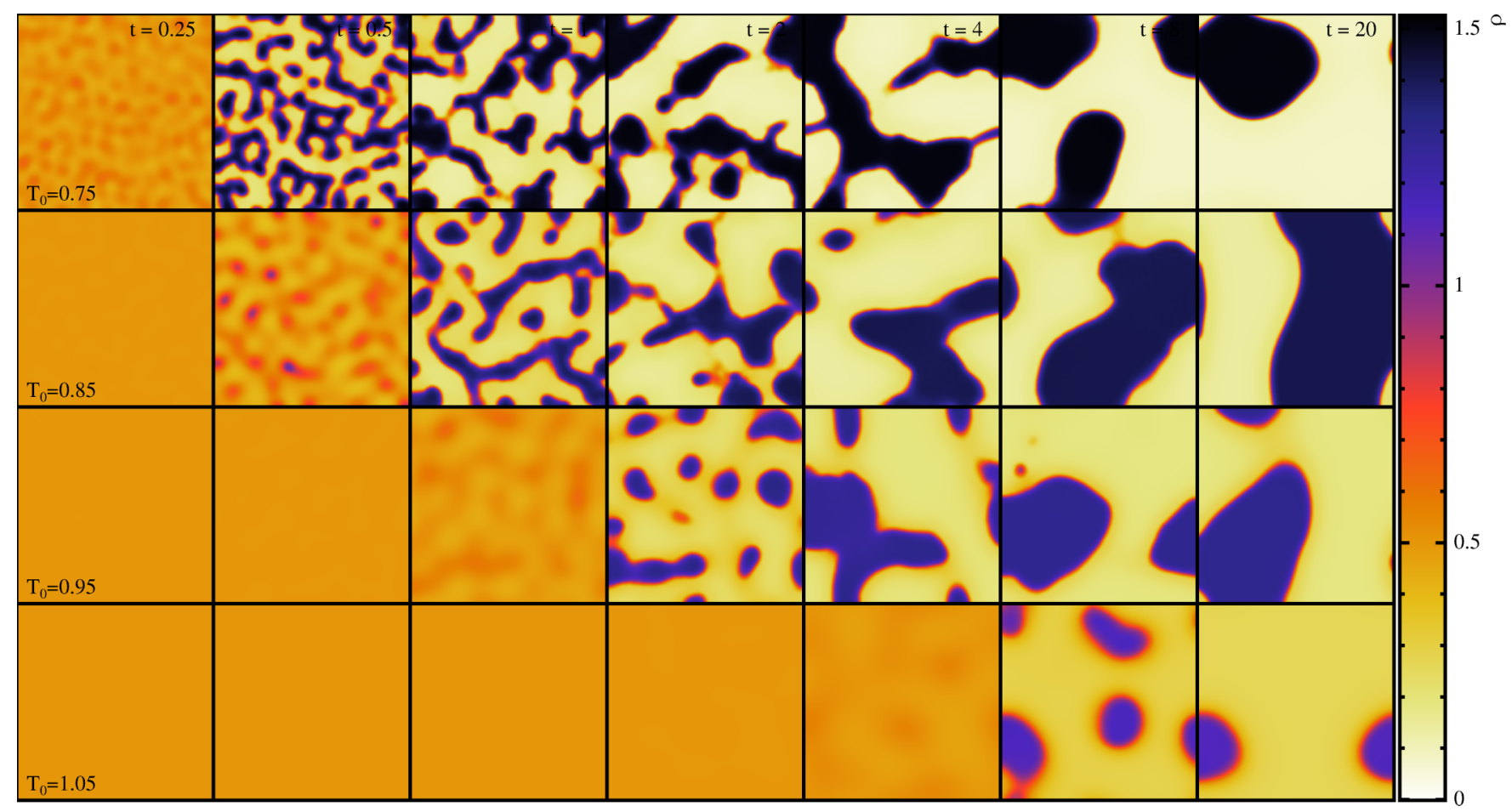

FIG. 6. (Color online) Comparison of density-rendered cross-section slices from simulation snapshots at the times $t=0.25,0.5,1.0,2.0$, 4.0, 8.0, and 20.0. Exemplarily the simulations with initial $T_{0}=0.75,0.85,0.95$, and 1.05 are shown (from top to bottom) with a side length of the box size $L=1$. Note that the actual shapes of the three-dimensional structures cannot be determined in the two-dimensional representation.

in correspondence with the prolonged dynamical evolution it is clearly visible that not only the number of initially growing nuclei is lowered with increasing temperature but also the wavelength of the fluctuations increase. Thus, the interfaces between separated phases become broader and more diffuse. In the simulations with $T_{0}=0.95$ and upwards the interfaces of actually separated liquid drops are found to be diffuse enough to overlap (e.g., Fig. 6 at $T_{0}=0.95$ and $t=20$ in the top left of the box). Moreover, the simulations suggest that high temperatures near $T_{c}$ (and therefore a long wavelength of the initial fluctuations and the increase in time of demixing) affect more of a nucleation-like demixing behavior than the SD. This phenomenon is also predicted by a theory presented in Refs. [1,5] and indeed already observed here at $T_{0}=1.1$. Such a formation of a spherical-shaped droplet is shown in Fig. 7. The cross-section is positioned at the center of the left droplet. At $t=40$ a stable droplet with a homogeneous density inside and a diffuse interface is formed. The main portion of kinetic energy of the system is in the interfaces. The fluid velocity vanishes beside the interfaces. A second droplet forms at the same time in a lower level of the box. It is barely identifiable in the density plot on the right-hand side of the box, but becomes visible in the temperature plot with velocity field vectors. The heating of the liquid and cooling of the vapor medium is also observed. In the further temporal evolution the temperature peak vanishes due to thermal conduction and results in a homogeneous temperature within the whole simulation volume. Also, the droplets do not remain static. At later times the nuclei grow and undergo coalescing processes as it does at deeper quenches.

The effects of the temperature field and thermostat shall now be studied in a more detailed manner. Therefore, the thermal evolution is plotted in Fig. 8 in the temperature-density phase diagram. Here the density and temperature values are also obtained as already described in the previous section. The dependency between quench depth and prolongation can be confirmed. The distances between the marked points at equidistant times with $\Delta t=0.005$ become shorter in the initial stage of separation with higher $T_{0}$. This rapid change in the density values of the separated phases is coupled to the temperature by the caloric vdW-EOS. Thus, the liquid phase is

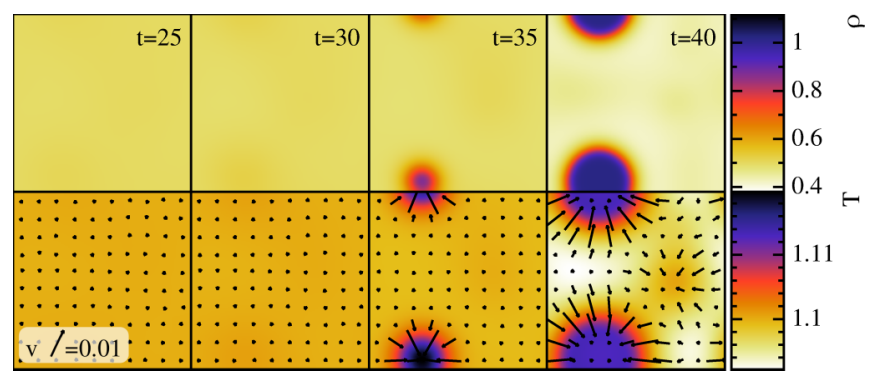

FIG. 7. (Color online) Nucleation in spinodal regime at a temperature $T=1.1$ near $T_{c}$. Time evolution of density-rendered (top) and temperature-rendered (bottom) cross-section slices. The vectors indicate the velocity field. 


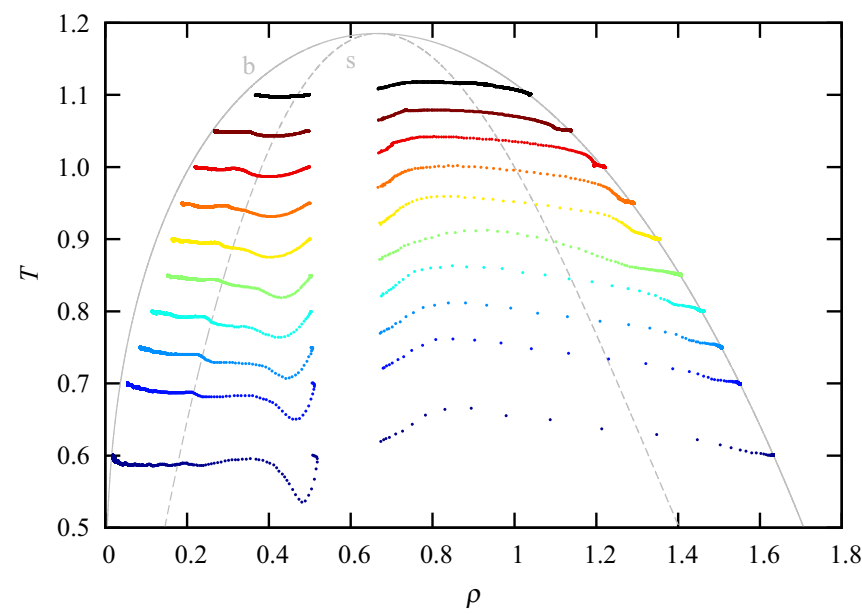

FIG. 8. (Color online) Temperature-density diagram at equidistant timesteps with $\Delta t=0.005$ and initial density $\rho_{0}=0.5$ for various temperatures [color coding as in Fig. 5, from $T_{0}=0.6$ (bottom) to 1.10 (top)]. The gray lines represent the binodal (solid line) and spinodal (dashed line) curves from vdW theory and Maxwell construction.

heated and the vapor phase is cooled. The faster the phase separation occurs and, hence, the density changes, the stronger the temperature deviations become. In the further evolution $\rho_{v}$ and $\rho_{l}$ continue to approach the binodal and the temperature deviations vanish. Since the thermostat only scales the $T_{i}$ by a factor [see Eq. (14)] in order to satisfy the condition $\bar{T}=T_{0}$, it cannot level out the thermal fluctuations. Hence, this process must be due to thermal conduction, which explains also why the temperature deviations are higher at deeper quenches. The effect of conduction becomes too weak to compensate the cooling and heating of the phases that occur as a result of the change in density.

In the late-stage time regime, when DG is dominant, latent heat is fully released and, therefore, the thermostat becomes less important for the demixing process. In Fig. 9 the characteristic lengths for the liquid $\xi_{l}$ are shown. Because the curves actually overlap, they are shifted by an exponential temperature-dependent factor for reasons of clarity and better comparability. Concerning the growth rates, it is found that the theoretical predicted LS mechanism is correct for the deeper quenched simulations. A time regime with a $\alpha=1 / 3$ scaling, right after the initial stage, can be clearly identified for the simulations with $T_{0}=0.6$ up to $T_{0}=0.95$. This $1 / 3$-scaling either ends due to finite-size effects (as it is for $T_{0}=0.6$ and $T_{0}=0.7$ ) or the $1 / 3$ exponent changes to a value slightly around $2 / 3$, which is in accordance with the theoretical predictions for IH growth. Our simulations show that the transition time $\tau_{2}$, that marks the crossover, depends on $T_{0}$. One possible suggested dependency for this transition is highlighted with the bold bar in Fig. 9. Even the slight kink in the $T_{0}=1.0$ curve may be due the crossover between the two growth regimes. For lower quench depths the growth rate of $2 / 3$ appears immediately after the initial stage. See Appendix for detailed listing of one possible realization of fit intervals and the corresponding fitted exponents.

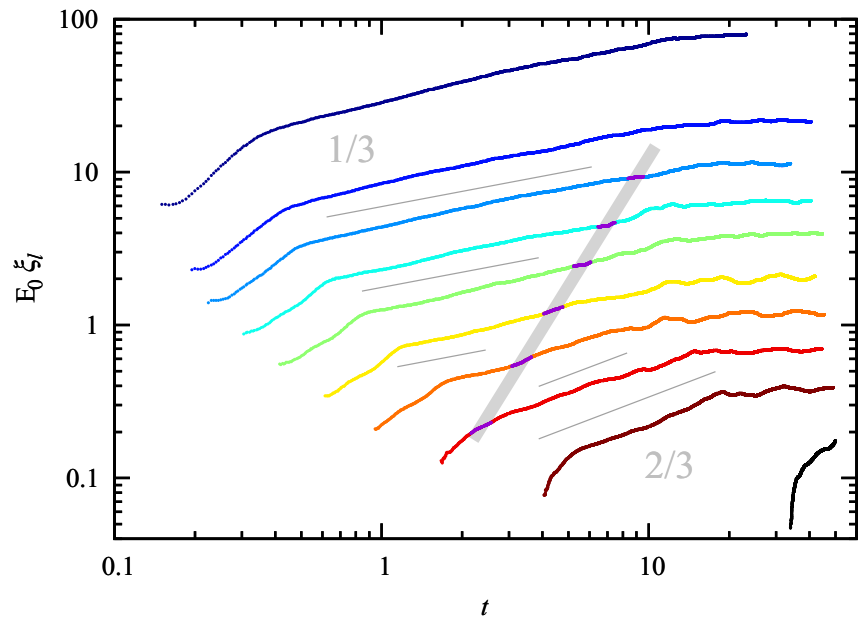

FIG. 9. (Color online) Log-log representation of the evolution of the characteristic lengths $\xi_{l}$ for various temperatures in time [color coding as in Figs. 5 and 8 , from $T_{0}=0.6$ (top) to 1.10 (bottom)]. For reasons of comparability the lengths are each shifted by a factor $E_{0}=\exp \left[\left(T_{c}-T_{0}\right) / 0.1\right]$ that depends on the initial $T_{0}$. The gray lines are guides to the eye and highlight the transitions from LS to IH growth (bold line) and the two growth rates of $1 / 3$ and $2 / 3$ (thin lines). Note that the growth of $\xi_{l}$ for deeper quenches is limited due to finite-size effects before the crossover to IH regime occurs.

This time dependency can also be interpreted with Fig. 6 in view. The domination of the hydrodynamic regime in the very late stage can be explained with the growth of $\xi_{l}$. It means that facing domains (actually still separated, but of the same phase) approach each other and coalescence becomes the dominant growth process over the coarsening. As it was mentioned above the width of the interfaces increases with increasing $T_{0}$. This might be understood in terms of surface tension $\sigma$, which is mainly responsible for the coarsening of the phases. A theoretical approach can already be derived from the vdW theory [29]. It takes the form $\sigma \approx\left(1-T / T_{c}\right)^{\gamma}$, where $\gamma \approx 11 / 9$ [54]. Hence, for higher temperatures the surface tension decreases. Several studies show the capability of the vdW-SPH method to capture the surface tension phenomenon well [30,31]. In addition, the theoretical expression for planar interfaces from vdW theory relates $\sigma$ to the thickness of the interface $L$, since it can be calculated by an integration of the square of the density gradient [55]. Based on the vdW theory, the density profile perpendicular to the interface can be described by a $\tanh (2 x / L)$ function and, therefore, the interface width diverges as $T$ approaches the critical temperature $T_{c}$ [56]. Moreover, the diffuse interfaces of separated domains overlap, such that they can interact hydrodynamically even if the drops are far away from each other. Thus, the interface thickness explains that the transition from LS to IH occurs earlier in time, and, therefore, depends on $T_{0}$. This transition point in time $\tau_{2}$ actually scales with the quench depth $\left(1-T_{0} / T_{c}\right)$. To this a least-square regression is shown in Fig. 10. As it was discussed above, the slope of the fitted curve should be determined by the mean distance between the separated droplets, i.e., taking the domain size and the interface width into account. Thus, changing the values of $\bar{a}$ or $\bar{b}$ would affect the interface widths and thus also 


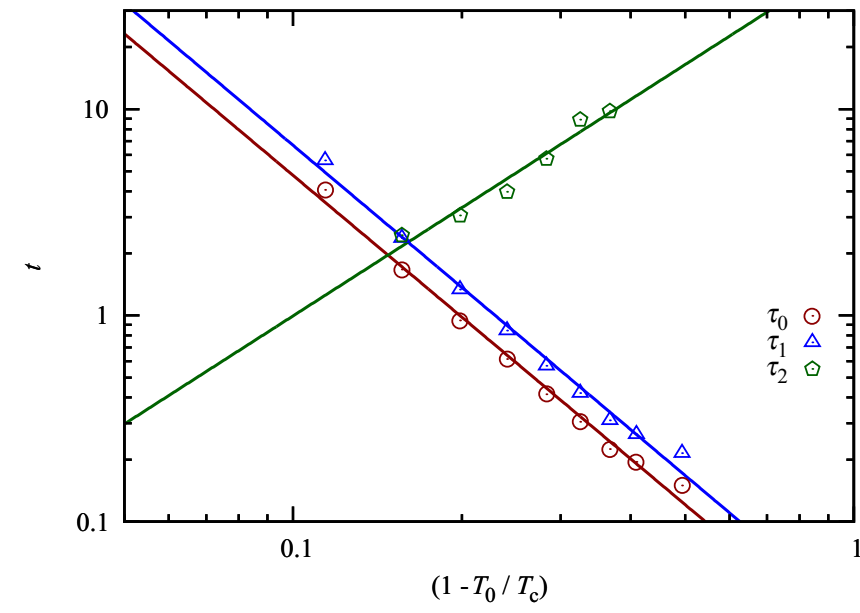

FIG. 10. (Color online) The points in time $\tau_{0}$ where $\rho$ first exceeds $\rho_{c}, \tau_{1}$ where $\xi_{v}$ have their minimum, and the points in time $\tau_{2}$ of the transition from LS to IH growth as functions of the quench depth. The solid lines correspond to least-square regression with the exponents $\alpha_{0}=-2.28 \pm 0.08, \alpha_{1}=-2.29 \pm 0.08, \alpha_{2}=$ $0.174 \pm 0.18$. The times for $T_{0}=1.1$ were excluded, because of its extraordinary nucleation-like growth behavior.

the slope of the transition line. But the question as to which quantity can affect this transition line to shift in time must be addressed to the thermal conductivity. It is responsible for the compensation of thermal fluctuations and the driving force for the LS mechanism. In accordance with the results from thermal LB simulation [28], a higher value of $\kappa$ is expected to reduce the duration of the SD regime and could affect a shift of the transition line.

Figure 10 shows also the scaling behavior of the times $\tau_{0}$ and $\tau_{1}$. It also supports the statement that the whole demixing process becomes slower with decreasing quench depth. The times of demixing $\tau_{0}$ and the point in time $\tau_{1}$, where $\xi_{v}$ reaches the minimum, can be fitted with an exponential growth (the simulation with $T=1.1$ was not taken into account because of its spherical non-SD-typical nucleation growth behavior). This is in agreement with the results that are obtained from deepquench simulations of binary mixtures [15] where a similar correlation is found.

For a final remark, the pure thermal simulation $\left(T_{0}=0.8\right.$, without thermostat) from Sec. IIIB1 is compared to the results that are obtained with different quench depths and applied thermostat. As it was already mentioned, the initial demixing progress (concerning the wavelength of "homophase fluctuations") is very similar to $T_{0}=0.8$ with thermostat. The differing further evolution might be due to the thermal heating. The comparison between Figs. 3 and 6 reveals a similarity to the $T_{0}=1.1$ simulation in a later stage. Indeed, the simulation without thermostat seems to cross between the thermostat simulations with the rise of temperature. That explains the observed $1 / 2$ exponent from Fig. 4(b). As it was already expected in the previous section, it is truly the result of an overlap between the $1 / 3 \mathrm{LS}$ growth, driven by the fluctuations that raised in the early time regime, and the $2 / 3 \mathrm{IH}$ exponent that occurs earlier in time for higher mean temperatures.

\section{CONCLUSION}

The SPH method in its modern formulation with extensions to vdW-EOS and thermal conduction is proved to capture the dynamics of phase transitions such as spinodal decomposition. This is even possible without complicated extensions such as density gradient forces or the separation of the vdW-EOS in short-range repulsive and long-range attractive components. Only minor improvements due to newest findings and developments to SPH such as the Wendland kernel and the thermostat are necessary. The internal energy and, therefore, also the thermal evolution is an inherent part of the method. Due to the massive parallelization algorithms the method provides the choice of very high resolutions (up to $\sim 10^{7}$ ). But in the context of phase separation it produces already satisfactory results with spatial and temporal resolutions that are even smaller by an order of magnitude than the resolution requirements for comparable mesoscopic simulation methods such as thermal-LB. Thus, the time range can be chosen much longer and the computational cost is reasonably low. A typical simulation run with $10^{5}$ particles and $T_{0}=0.8$ up to a time $t=50$ is performed on $32 \mathrm{CPU}$ and lasts about $48 \mathrm{~h}$, and, thus the complete calculation of the initial SD regime at $t \approx 1$ is completed within $1 \mathrm{~h}$.

The correctness of the SPH interpretations of the thermal conduction equation and the newly developed scaling thermostat are verified with a number of validation test cases with both static and dynamic tests. The static test has shown that the thermostat corrects temperature deviations that arise by density estimation errors. The dynamical test reveals that for sensible results an adequate resolution must be chosen.

The dynamics of spinodal decomposition are studied both with a pure thermal simulation and with a scaling thermostat. It is found that "spongelike" structures are formed in three dimensions. The pure thermal evolution is characterized by an increase in temperature in the initial stage due to latent heat. The growth rates are in accordance with the observations from the thermostat simulations. The initial quench depth is expected to determine the intensity of thermal fluctuations and, thus, the duration of the diffusive LS growth. With the increase in temperature, the interfaces broaden and $\mathrm{IH}$ growth becomes dominant. Therefore, a transition growth rate between the two regimes is found. This is expected to result from an overlap of the two growth rates. Although the pure thermal evolution seems more realistic with regard to experiments, this temporal overlapping of the growth regimes makes it more difficult to clarify the physical origins of the growth processes. But the comparison with simulations with various initial temperatures and applied thermostat supports the hypothesis of overlap. These simulations confirm the dependency between temperature and prolongation in the initial stage. The initial demixing is found to be strongly correlated with heating and cooling of the phases, where the deviations from the mean temperature become stronger if the quench depth increases. A crossover (which is also theoretically predicted) between the two growth mechanism (LS and IH) in the late-stage time regime is observed for several initial temperatures. Moreover, it is found that the time of crossover increases with increasing the quench depth and follows a scaling law. Thus, deep 
quenches show pure LS and low quenches pure IH growth. The line of transition might be shifted in time with changing the thermal conductivity. This is in accordance with results from thermal-LB simulations. For very low quenches (near the critical temperature) the dynamic becomes slower and the separation process is rather more nucleationlike than $\mathrm{SD}$, which is also in accordance with theoretical predictions. We note that the described SPH method also produces SDnucleation even at higher temperatures than here presented, but therefore the initial density should be set to a value below the spinodal.

For future works there are several appropriate investigations. For the reason of a better comparability with experimental results we expect that an extended thermostat model that allows finite quench rates will be straightforward. In this work we observe a correlation between the hydrodynamic interaction range and the interface width. We did not focus on it in detail, although it would improve the understanding of the role of hydrodynamics. But for this purpose it is rather reasonable to use a more simple model and to study therein the fluid flow and temperature field evolution of the late-stage IH regime. One possible model could be the coalescence of actually equilibrated liquid droplets in a vapor medium and vice versa. The vdW-SPH method is also a promising candidate for sensible dynamic simulations of evaporation phenomenons like hydrodynamic cavitation and jet formation.

\section{ACKNOWLEDGMENTS}

The authors gratefully acknowledge the computing time granted by the John von Neumann Institute for Computing (NIC) and provided on the supercomputer JUROPA at Jülich Supercomputing Centre (JSC). Providing computing resources on the Scientific Computing Cluster (SCC) by the University of Konstanz is also gratefully acknowledged. For the rendered plots we used SPLASH [57], a visualization tool for SPH data.
TABLE II. The exponents $\alpha$ (with asymptotic standard error ${ }^{\mathrm{a}} \delta \alpha$ ) are least-square fitted and refer to the characteristic lengths for the liquid phase presented in Fig. 9 and their corresponding lengths for the vapor phase.

\begin{tabular}{llccc}
\hline \hline$T_{0}$ & Time & $\alpha_{l} \pm \delta \alpha_{l}$ & Time & $\alpha_{v} \pm \delta \alpha_{v}$ \\
\hline 0.60 & $0.40-9.00$ & $0.365 \pm 0.001$ & $0.40-9.20$ & $0.390 \pm 0.002$ \\
0.70 & $0.47-10.00$ & $0.352 \pm 0.001$ & $0.47-10.20$ & $0.379 \pm 0.001$ \\
0.75 & $0.55-8.70$ & $0.333 \pm 0.001$ & $0.55-8.70$ & $0.403 \pm 0.001$ \\
& $10.7-13.7$ & $0.443 \pm 0.002$ & - & - \\
0.80 & $0.65-7.00$ & $0.329 \pm 0.001$ & $0.65-7.00$ & $0.424 \pm 0.001$ \\
& $7.01-10.9$ & $0.671 \pm 0.006$ & $7.01-9.90$ & $0.621 \pm 0.004$ \\
0.85 & $0.81-5.70$ & $0.404 \pm 0.001$ & $0.80-5.30$ & $0.442 \pm 0.001$ \\
& $8.6-11.90$ & $0.659 \pm 0.004$ & - & - \\
0.90 & $1.20-3.6$ & $0.417 \pm 0.002$ & $1.20-9.60$ & $0.482 \pm 0.002$ \\
& $3.90-5.40$ & $0.619 \pm 0.002$ & - & - \\
0.95 & $1.70-3.10$ & $0.386 \pm 0.003$ & $1.72-10.00$ & $0.487 \pm 0.002$ \\
& $3.11-7.2$ & $0.582 \pm 0.002$ & - & - \\
1.00 & $2.90-6.40$ & $0.652 \pm 0.002$ & $2.9-8.70$ & $0.617 \pm 0.001$ \\
1.05 & $6.00-14.0$ & $0.687 \pm 0.002$ & $6.00-16.5$ & $0.766 \pm 0.001$ \\
\hline \hline
\end{tabular}

${ }^{a}$ Asymptotic standard error expresses the accuracy in accordance to the calculated characteristic lengths.

\section{APPENDIX: FIT INTERVALS AND EXPONENTS}

In Fig. 9 only the characteristic lengths for the liquid phase $\xi_{l}$ are presented, whereas the $\xi_{v}$ are not shown. Moreover, the crossover between the different growth regimes is only schematically illustrated. For completeness we present in Table II one possible choice of fit intervals and the corresponding fitted exponents for both the liquid and the vapor phase. Note that the suggested time intervals represent only one possible realization. Therefore, the fitted exponents $\alpha$ are also just suggestions. In Fig. 11 the fit intervals and the corresponding growth rates are highlighted on the characteristic lengths for (a) the liquid and (b) the vapor phases.
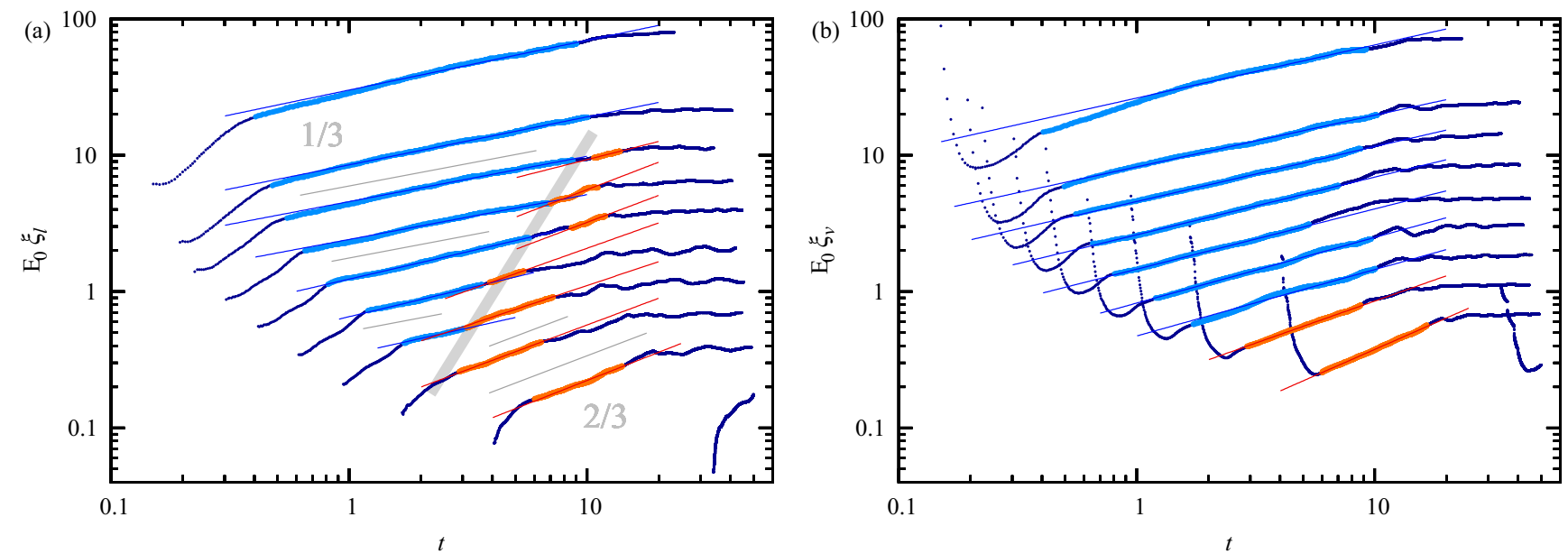

FIG. 11. (Color online) Log-log representation of the evolution of the characteristic lengths for the temperatures $T_{0}=0.60,0.70,0.75$, $0.80,0.85,0.90,0.95,1.00,1.05$, and 1.10 (from top to bottom) for (a) the liquid phase $\xi_{l}$ and (b) the vapor phase $\xi_{v}$. The curves are also shifted as described in the legend for Fig. 9. The fit intervals from Table II are each highlighted by enlarged (lighter) points and the corresponding fitted exponents are shown with straight lines. 
[1] K. Binder, Rep. Prog. Phys. 50, 783 (1987).

[2] J. W. Cahn and J. E. Hilliard, J. Chem. Phys. 28, 258 (1958).

[3] J. W. Cahn, Acta Metall. 9, 795 (1961).

[4] J. W. Cahn, J. Chem. Phys. 42, 93 (1965).

[5] K. Binder, Phys. Rev. A 29, 341 (1984).

[6] Y. Gan, A. Xu, G. Zhang, Y. Li, and H. Li, Phys. Rev. E 84, 046715 (2011).

[7] H. Furukawa, Adv. Phys. 34, 703 (1985).

[8] A. J. Bray, Adv. Phys. 43, 357 (1994).

[9] I. M. Lifshitz and V. V. Slyozov, J. Phys. Chem. Solids 19, 35 (1961).

[10] E. D. Siggia, Phys. Rev. A 20, 595 (1979).

[11] H. Furukawa, Physica A 204, 237 (1994).

[12] F. J. Alexander, S. Chen, and D. W. Grunau, Phys. Rev. B 48, 634 (1993).

[13] R. Yamamoto and K. Nakanishi, Phys. Rev. B 49, 14958 (1994).

[14] H. Tanaka and T. Araki, Phys. Rev. Lett. 81, 389 (1998).

[15] N. Vladimirova, A. Malagoli, and R. Mauri, Phys. Rev. E 58, 7691 (1998).

[16] P. B. Warren, Phys. Rev. Lett. 87, 225702 (2001).

[17] P. B. Warren, Phys. Rev. E 68, 066702 (2003).

[18] A. G. Lamorgese and R. Mauri, Phys. Fluids 21, 044107 (2009).

[19] A. Winkler, P. Virnau, K. Binder, R. G. Winkler, and G. Gompper, J. Chem. Phys. 138, (2013).

[20] M. Hirschler, M. Huber, W. Saeckel, P. Kunz, and U. Nieken, Math. Prob. Eng. 2014, 694894 (2014).

[21] W. R. Osborn, E. Orlandini, M. R. Swift, J. M. Yeomans, and J. R. Banavar, Phys. Rev. Lett. 75, 4031 (1995).

[22] K. R. Mecke and V. Sofonea, Phys. Rev. E 56, R3761 (1997).

[23] V. Sofonea, A. Lamura, G. Gonnella, and A. Cristea, Phys. Rev. E 70, 046702 (2004).

[24] P. K. Jaiswal, S. Puri, and S. K. Das, Phys. Rev. E 85, 051137 (2012).

[25] P. K. Jaiswal, S. Puri, and S. K. Das, Europhys. Lett. 97, 16005 (2012).

[26] S. Roy and S. K. Das, Phys. Rev. E 85, 050602 (2012).

[27] S. Roy and S. K. Das, Soft Matter 9, 4178 (2013).

[28] Y. Gan, A. Xu, G. Zhang, P. Zhang, and Y. Li, Europhys. Lett. 97, 44002 (2012).

[29] J. van der Waals, J. Stat. Phys. 20, 200 (1979).

[30] S. Nugent and H. A. Posch, Phys. Rev. E 62, 4968 (2000).

[31] Leonardo Di G. Sigalotti, J. Troconis, E. Sira, F. Peña Polo, and J. Klapp, Phys. Rev. E 90, 013021 (2014).
[32] Y. Meléan, L. D. G. Sigalotti, and A. Hasmy, Comput. Phys. Commun. 157, 191 (2004).

[33] Y. Meléan and L. D. G. Sigalotti, Int. J. Heat Mass Trans. 48, 4041 (2005).

[34] A. Tartakovsky and P. Meakin, Phys. Rev. E 72, 026301 (2005).

[35] H. López and Leonardo Di G. Sigalotti, Phys. Rev. E 73, 051201 (2006).

[36] A. M. Tartakovsky, K. F. Ferris, and P. Meakin, Comput. Phys. Commun. 180, 1874 (2009).

[37] Z. Xu, P. Meakin, and A. M. Tartakovsky, Phys. Rev. E 79, 036702 (2009).

[38] K. Szewc, J. Pozorski, and J.-P. Minier, Int. J. Multi. Flow 50, 98 (2013).

[39] X. Yang, M. Liu, and S. Peng, Comput. Fluids 92, 199 (2014).

[40] V. Springel, Annu. Rev. Astron. Astrophys. 48, 391 (2010).

[41] D. J. Price, J. Comput. Phys. 231, 759 (2012).

[42] D. Sijacki and V. Springel, Mon. Not. R. Astron. Soc. 371, 1025 (2006).

[43] L. B. Lucy, Astron. J. 82, 1013 (1977).

[44] R. A. Gingold and J. J. Monaghan, Mon. Not. R. Astron. Soc. 181, 375 (1977).

[45] F. Bürzle, P. C. Clark, F. Stasyszyn, T. Greif, K. Dolag, R. S. Klessen, and P. Nielaba, Mon. Not. R. Astron. Soc. 412, 171 (2011).

[46] V. Springel, Mon. Not. R. Astron. Soc. 364, 1105 (2005).

[47] W. Dehnen and H. Aly, Mon. Not. R. Astron. Soc. 425, 1068 (2012).

[48] P. W. Cleary and J. J. Monaghan, J. Comput. Phys. 148, 227 (1999).

[49] M. Jubelgas, V. Springel, and K. Dolag, Mon. Not. R. Astron. Soc. 351, 423 (2004).

[50] J. J. Monaghan, J. Comput. Phys. 136, 298 (1997).

[51] D. Frenkel and B. Smit, Understanding Molecular Simulation: From Algorithms to Applications, Vol. 1 (Academic Press, San Diego, 2001).

[52] O. Kum and W. Hoover, J. Stat. Phys. 76, 1075 (1994).

[53] L. D. Landau and E. M. Lifshitz, Fluid Mechanics: Course of Theoretical Physics, Vol. 6 (Elsevier, New York, 2013).

[54] E. A. Guggenheim, J. Chem. Phys. 13, 253 (1945).

[55] R. Lovett, P. W. DeHaven, J. J. Vieceli, and F. P. Buff, J. Chem. Phys. 58, 1880 (1973).

[56] E. S. Wu and W. W. Webb, Phys. Rev. A 8, 2065 (1973).

[57] D. J. Price, PASA 24, 159 (2007). 